\title{
Life-threatening Cardiac Failure: A Rare Complication of Branchial Cleft Cyst
}

\author{
${ }^{1}$ Shi Da Felix Yam, ${ }^{2}$ Tak Lit Derek Fung, ${ }^{3}$ Lap Chiu Donald Tang
}

\begin{abstract}
Branchial cleft cyst is a well-known head and neck anomaly. Patients commonly presented with cosmetic problems. Complications including pressure symptoms, pain and superimposed infection have been reported. Rarely, it could present with carotid sinus syndrome.
\end{abstract}

Case report: We reported a case who presented with vasovagal syncope and cardiogenic shock was found to have huge branchial cleft cyst. Carotid sinus syndrome secondary to compression by the branchial cyst was suspected. It was managed with inotropic support and needle decompression. She later readmitted for superimposed infection which was treated by antibiotics. Surgical excision was performed to render her complete cure. This was the second reported case of a benign branchial cleft cyst causing cardiac compromise in the literature.

Conclusion: Benign branchial cleft cyst may present with lifethreatening carotid sinus syndrome. Timely needle decompression should be performed for temporary relieve and definitive surgery is required for cure.

Keywords: Branchial cleft cyst, Carotid sinus syndrome, Branchial anomaly, Bradycardia, Branchial cyst complication.

How to cite this article: Yam SDF, Fung TLD, Tang LCD. Lifethreatening Cardiac Failure: A Rare Complication of Branchial Cleft Cyst. Int J Head Neck Surg 2014;5(3):158-160.

Source of support: Nil

Conflict of interest: None

\section{INTRODUCTION}

Branchial cleft cyst is included under the category of branchial anomalies, which is composed of a heterogenous group of congenital malformations that arise from incomplete obliteration of pharyngeal clefts and pouches during embryogenesis. Branchial cleft anomalies are the second most common congenital head and neck lesions and account for $30 \%$ of all congential head and neck lesions, with second branchial cleft lesions accounting for $95 \%$ of all branchial cleft malformations. ${ }^{2}$ Complications including pressure symptoms, pain and superimposed infection have been reported. ${ }^{1}$ Rarely, it can present with a life-threatening condition. Knowing the uncommon presentation and the acute management could avoid

\footnotetext{
${ }^{1,2}$ Resident, ${ }^{3}$ Head

${ }^{1-3}$ Department of Surgery, Queen Elizabeth Hospital, Hong Kong
}

Corresponding Author: Tak Lit Derek Fung, Resident Department of Surgery, Queen Elizabeth Hospital, Hong Kong Phone: 98304916, e-mail: derekfungt|@gmail.com potential tragedy. In this report, we described a patient who presented with cardiogenic shock secondary to compression on the carotid sinus.

\section{CASE REPORT}

A 30-year-old Indonesian, with known history of left neck mass, was admitted for micturition syncope. Ultrasound done 2 months ago in another hospital revealed a left neck cystic lesion around $11 \mathrm{~cm}$ in length, which extended cranially beyond carotid bifurcation and caudally into subclavicular region. Physical examination revealed a huge and tense cystic lesion over the left side of the neck, with tracheal deviation to the right. Chest X-ray showed patent trachea and clear chest fields. She complained of shortness of breath after admission and developed an episode of hypotension in emergency room which responded to fluid challenge. Her oxygen saturation was maintained at $100 \%$ with oxygen supplement via nasal cannula. After admission, her sensorium gradually deteriorated. She was drowsy but was still responsive to verbal stimulant. Cardiac monitor showed bradycardia down to around 35 to 55 beats per minute (bpm) and hypotension with systolic blood pressure lower than $90 \mathrm{~mm} \mathrm{Hg}$. Electrocardiography (ECG) revealed junctional bradycardia. Dopamine infusion was started to maintain adequate organ perfusion. The underlying cause was suspected to be due to an increase in vagal tone secondary to compression of carotid sinus. Urgent computed tomography (CT) scan revealed a large unilocular cystic lesion in left side of neck, radiologically compatible with a branchial cyst, which narrowed the common carotid artery (Figs 1 and 2). Another superior anterior mediastinal lesion was seen, abutting the inferomedial aspect of the aforementioned cystic lesion, containing fat and solid enhancing component and was suspected to be dermoid or teratoma. Needle decompression was performed, yielding dark greenish fluid. The pulse rate improved dramatically after needle aspiration while the hypotension required 2 days to wean off the inotropes. Her blood pressure returned to normal range and her baseline pulse rate was around 70 to $80 \mathrm{bpm}$. Twenty-four hour ECG which was later arranged revealed no abnormality. Aspirated fluid was negative for both bacterial culture and malignancy. She was readmitted 2 weeks 


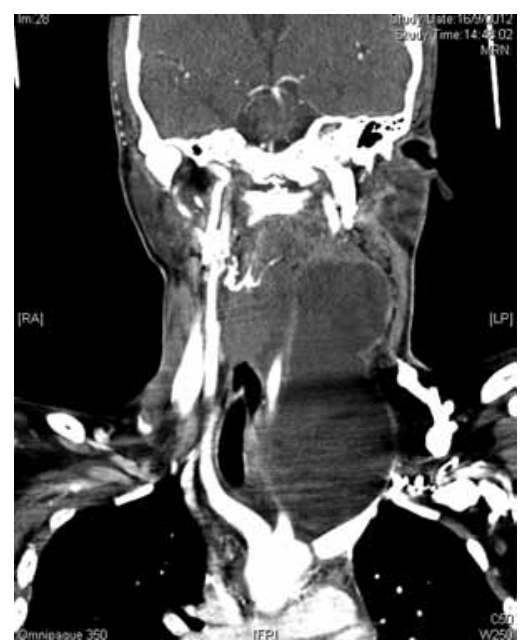

Fig. 1: Coronal view of the branchial cyst on CT scan showing compression on the carotid sinus

later for fever and recurrence of left neck swelling after CT-guided biopsy of the mediastinal mass. Repeated CT scan showed increase in enhancement of the mediastinal mass. She was managed as superimposed infection with antibiotics. Resection of the branchial cyst and superior mediastinal mass was done after the infection was well controlled, and the patient recovered uneventfully. Final pathology was 'cystic lesion' (which was compatible with but not diagnostic of branchial cyst) for the cystic component and 'mature teratoma' for the mediastinal mass. She had no recurrence of neck swelling or syncope since then.

\section{DISCUSSION}

Our patient presented with a second branchial anomaly. It was a type II branchial cleft cyst according to Bailey's classification. ${ }^{3}$ It lay adjacent to the carotid sheath yet did not pass through between the external and internal carotid artery as in type III. When enlarged, the cyst could affect the cardiovascular system through narrowing of the artery and applying pressure on the carotid sinus.

Carotid sinus syndrome, also known as carotid sinus hypersensitivity, is an exaggerated response of the carotid sinus to manual stimulation. It could cause bradycardia or vasodilatation, resulting in hypotension, pre-syncope or syncope. Classical presentation is that patient may faint while shaving. Carotid sinus contains baroreceptors which detect changes in stretch and transmural pressure. Decrease in arterial pressure leads to a decrease in receptor firing and thus afferent impulses to the medulla. Consequently, this leads to decrease in vagal tone and sympathetic firing to the heart and blood vessels, causing cardiac inhibition or vasodilatation. The baroreceptors reflex also includes endocrine reflexes, which include renin and anterior pituitary hormones. ${ }^{4}$

Baroreceptors adapt to prolonged stretch. The adaptation or resetting, begins to occur within minutes of a

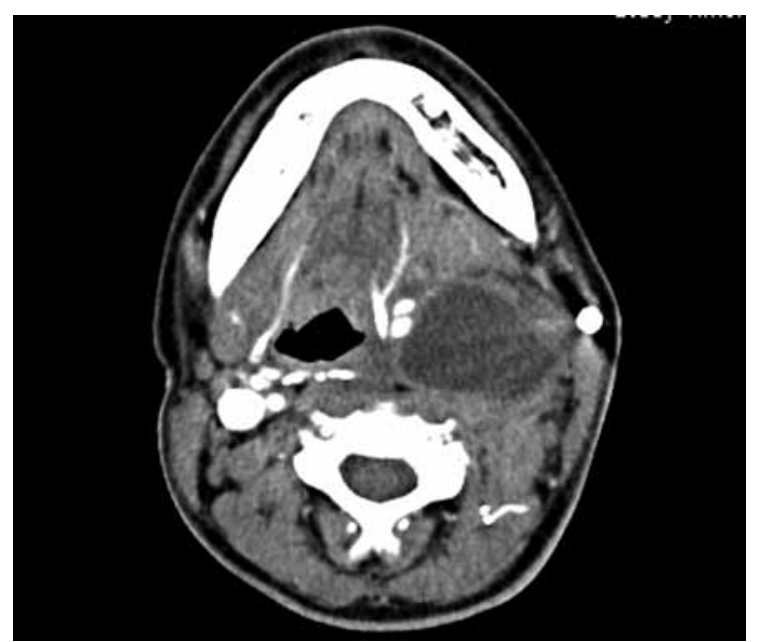

Fig. 2: Transverse view of the branchial cyst on CT scan at the level of carotid bifurcation

sustained change in blood pressure. Thus, baroreceptor afferent firing codes acute changes in pressure rather than a specific pressure. ${ }^{4}$ It is, therefore, noted that chronic compression of the carotid sinus by the branchial cyst should rarely cause a significant cardiovascular effect. In our case, our patient did not experience any problem for months with such a huge cyst. However, symptoms arose on a day when there was a sudden increase of the size of the cyst. The sudden enlargement could be due to hemorrhage or infection. What if the cyst enlarged by a gradual manner? Could it still cause carotid sinus syndrome? The answer could still be yes. There are reports of carotid sinus syndrome secondary to head and neck tumors. ${ }^{5}$ Different mechanisms due to progressive and prolonged compression by tumor were postulated. ${ }^{6}$ Hence for any head and neck mass closely related to carotid sinus, we should be alert for such potential complication.

A different rate of recovery for pulse rate and blood pressure was observed in our case. We hypothesized that after needle decompression, the bradycardia component was immediately corrected as it was mainly controlled by the neural route. In contrast, the vasodepressor component, which involved endocrine response, required a longer time to recover.

The first case of branchial cleft cyst causing carotid sinus bradycardia was reported by Alexander et $\mathrm{al}^{7}$ in 2009. A 54-year-old man presented with a $5 \mathrm{~cm}$ left neck mass with associated pain, fatigue and episodic bradycardia down to $25 \mathrm{bpm}$. The mass was initially drained repeatedly with temporary improvement followed by recurrence over one and a half years. Subsequently, it was decided to excise the mass after the patient received cardiac clearance. Thus, a pacemaker was implanted which somewhat improved the fatigue. However, resolution of both bradycardia and fatigue was achieved 
only after the mass was excised. Our patient's symptoms resolved after aspiration. Repeated aspiration could be an option. However, repeated aspiration may lead to recurrence and superimposed infection as illustrated in our case. This is hence not a good option. Our patient was totally asymptomatic after surgery. Surgical excision is hence mandatory for definite cure and prevention of life-threatening hemodynamic effect.

\section{CONCLUSION}

Despite the benign nature of branchial cleft cysts, this case should alert clinicians that hypotension and syncope could be one of its rare complications and may be life-threatening as well. Lesions in close proximity to the carotid sinus should be intervened early. In case of cardiac compromise arise, inotropic support and timely needle decompression is recommended for temporary relieve before definitive surgery.

\section{REFERENCES}

1. Chandler JR, Mitchell B. Branchial cleft cysts, sinuses, and fistulas. Otolaryngol Clin North Am 1981 Feb;14(1):175-186.

2. Bajaj $Y$, Ifeacho $S$, Tweedie D, Jephson CG, Albert DM, Cochrane LA, et al. Branchial anomalies in children. Int J Pediatr Otorhinolaryngol (Comparative Study) 2011 Aug; 75(8):1020-1023.

3. Bailey H. The clinical aspects of branchial cysts. Br J Surg 1922;10(40):565-572.

4. Sved AF. Blood pressure: baroreceptors. In: Squire LR, editor. Academic Press, Oxford; 2009.

5. Hong AM, Pressley L, Stevens GN. Carotid sinus syndrome secondary to head and neck malignancy: case report and literature review. Clin Oncol (R Coll Radiol). Case Reports Review 2000;12(6):409-412.

6. Muntz HR, Smith PG. Carotid sinus hypersensitivity: a cause of syncope in patients with tumors of the head and neck. Laryngoscope. Case Reports 1983 Oct;93(10):1290-1293.

7. Alexander AA, Groblewski JC, Davidson BJ. Branchial cleft cyst causing carotid sinus syndrome. Arch Otolaryngol Head Neck Surg (Case Reports) 2009 Oct;135(10):1045-1047. 